\title{
Chronic Ulcerative Herpes Simplex Virus Infection of the Vulva
}

\author{
Kelly Griffith-Bauer ${ }^{a} \quad$ Mary O'Hearn ${ }^{b}$ Benjamin D. Ehst ${ }^{a}$ \\ Departments of ${ }^{\mathrm{a}}$ Dermatology and ${ }^{\mathrm{b}}$ Medicine, Oregon Health \& Science \\ University, Portland, Oreg., USA
}

\section{Key Words}

Chronic herpes simplex virus $\cdot$ HIV $\cdot$ Immune reconstitution inflammatory syndrome

\begin{abstract}
Herpes simplex virus infections in HIV-infected individuals can be clinically unusual and difficult to treat due to underlying problems with cell-mediated immunity and the occurrence of antiviral resistance. Additionally, partial or incomplete restoration of immune function may result in chronic ulcerations that require rotational treatments. In this report, we describe the case of a 38-year-old HIV-positive woman who developed the ulcerative form of chronic herpes simplex infection despite highly active antiretroviral therapy and valacyclovir prophylaxis. Repeated intravenous courses of foscarnet and topical cidofovir finally controlled her erosions as her cell-mediated immunity was slowly restored. This case highlights the challenges that still exist in diagnosing and managing this rare presentation of herpes simplex virus
\end{abstract}

\section{Introduction}

Mucocutaneous herpes simplex virus (HSV) infections are among the most common sexually transmitted diseases in HIV-infected individuals [1]. Typical acyclovirresponsive vesicular outbreaks as seen in HIV-uninfected individuals are the norm, although severity and number of recurrences can increase when host immunity is poor [2]. Additionally, classic outbreaks of genital HSV also increase after starting highly active antiretroviral therapy (HAART) as part of the well-described immune reconstitution inflammatory syndrome (IRIS) [3]. Uncommonly, HSV infections may become chronic and recalcitrant to treatment. These persistent infections are rare in the literature but have recently been grouped into ulcerative and pseudo-tumoral (hypertrophic or granulomatous) variants [4,5]. While increased acyclovir resistance is a contributing factor, poorly understood complications and/or defects during and after 
immune recovery are other likely contributors to chronic outbreaks. We report a case of chronic ulcerative HSV infection of the vulva in a patient with HIV developing almost 1 year after initiating HAART and continuing even after significant improvement in her CD4 cell count. This emphasizes the fact that these chronic HSV recurrences may start or persist outside of the normal 6-month window of IRIS and despite partial or complete restoration of immunity.

\section{Case Report}

A 38-year-old HIV-1-infected African American woman presented in September 2009 with several weeks of a painful vulvar ulcer. Her HIV disease was diagnosed in 2003 and she had been on HAART (lamivudine, zidovudine and atazanavir) since 2006, although with poor compliance. After another lapse in treatment for 1 year, she resumed therapy in 2008 with a prompt improvement in immune status 2 months later (viral load drop from $>1,000,000$ to 390 copies/ml and concomitant rise in CD4 count from 3 to $64 / \mathrm{mm}^{3}$ ). Her HAART regimen was changed to emtricitabine, tenofovir, atazanavir and ritonavir, and approximately 8 months later (11 months after resuming HAART) she developed the painful erosion on the inner left labia minora. Her viral load was still detectable at $240 \mathrm{copies} / \mathrm{ml}$ and her CD4 count had risen to $194 / \mathrm{mm}^{3}$. She had a history of genital herpes simplex and was on valacyclovir $500 \mathrm{mg}$ twice-daily prophylaxis up to this time with no clinical symptoms. Lesional culture did not detect HSV but she experienced some symptomatic relief with empiric increase in her valacyclovir dosing to thrice daily. However, the erosion persisted and by 6 months later had enlarged to involve both labia with painful ulceration and yellowish adherent exudates (fig. 1). HAART therapy was again changed to abacavir, lamivudine, atazanavir and ritonavir due to nausea. On this regimen, viral activity dropped to $<50$ copies/ml with a rise in CD4 count to $265 / \mathrm{mm}^{3}$. Again HSV was not detected on culture so a biopsy was obtained to confirm the suspicion and exclude the possibility of fixed drug reaction, erosive lichen planus, syphilis, or malignancy. The result was non-diagnostic, revealing diffuse mixed dermatitis with neutrophils, plasma cells, and numerous eosinophils. Immunohistochemistry did not detect HSV-1 or -2, and special stains for spirochetes were negative. The rapid plasma reagin was non-reactive as well. Supportive care with triamcinolone $0.1 \%$ ointment and topical lidocaine were started, and valacyclovir was continued. With spread of the ulceration to the perineal region 2 months later, another skin biopsy was performed showing similar findings (fig. 2), but the presence of HSV was confirmed by immunohistochemistry (fig. 3). IgG antibodies to HSV$1 /-2$ were detected at high titer in the serum confirming past exposure to HSV. There was no evidence of treponemal organisms and the rapid plasma reagin was again negative, no cytomegalovirus was detected by immunohistochemistry or PCR, and direct immunofluorescence was negative.

Acyclovir-resistant HSV was assumed and intravenous foscarnet was initiated at $40 \mathrm{mg} / \mathrm{kg}$ twice daily. After 3 weeks, her vulvar lesions had completely resolved and her perineum was greatly improved. Topical cidofovir $1 \%$ gel was then started twice daily with prompt worsening, so she resumed foscarnet for another 4 weeks. Ulcerations persisted again, so her dose of foscarnet was increased to $60 \mathrm{mg} / \mathrm{kg}$ twice daily for an additional 3 weeks. At follow-up, she showed improvement; however, she still had small persistent erosions. Her total CD4 count was still low at $168 / \mathrm{mm}^{3}$. She then resumed topical cidofovir and had slow but gradual improvement over the next 9 months. Valacyclovir prophylaxis was added and by 5 months later she was almost completely healed and comfortable. Her total CD4 count was now at $330 / \mathrm{mm}^{3}$. She continues to maintain control with this regimen.

\section{Discussion}

HSV infections are a common cause of morbidity in individuals infected with HIV and occur throughout the course of their infection and as part of immune reconstitution. Chronic HSV ulcers (those lasting more than 4 weeks) on the contrary had been primarily a consequence of advanced HIV/AIDS, yet the introduction of HAART has not completely abolished their occurrence [6]. This case is another example of the 
ulcerative subtype of chronic genital HSV and is similar to the few other cases of chronic HSV reported in the literature that develop during IRIS or thereafter [7-11]. Similar to those patients reported by Fox et al. [7], our patient developed severe ulcerative genital HSV, which was difficult to culture and detect, and poorly responsive to acyclovir derivatives, foscarnet and cidofovir. Like 1 patient in that report and the cases reported by Yudin and Kaul and Lanzafame, our patient clearly fit outside the normal window for IRIS [7-9].

The mechanism behind chronic HSV outbreaks is not known. Antiviral resistance certainly plays a role as suggested by a recent study where 5 of 7 cases of chronic HSV had clinical and in vitro resistance to acyclovir, cidofovir and/or foscarnet [4]. However, regardless of the switch to different therapies or the initial lack of antiviral resistance, all of those cases still took months to years to resolve. Antiviral resistance could not be proven in our patient as the virus was not cultured, but her poor response to repeated courses and dosage increases of foscarnet might suggest otherwise, since HSV is reported to heal, on average, within 6 days after the start of foscarnet treatment $[12]$.

Given the presence of an inflammatory infiltrate in chronic HSV lesions, another possible mechanism to explain persistence is that insufficient immunity to suppress viral activity occurs resulting in an imbalance in pro- and anti-inflammatory pathways akin to a chronic wound $[7,8]$. This is similar to the initial exaggerated immune responses that characterize IRIS, but the process is delayed in individuals that fail to rapidly or fully restore immunity. Our patient's CD4 count remained below $300 / \mathrm{mm}^{3}$ throughout her protracted course, and was beginning to heal only once her counts improved further. An interesting feature of the histopathology in this case was the unusual prominence of plasma cells and eosinophils not normally seen in recurrent HSV outbreaks [13]. This was also reported in the cases by Fox et al. [7] and may be underappreciated as many of the reports on chronic HSV noted herein and in the literature do not clearly describe the histologic infiltrates. Whether or not this finding may have relevance to our understanding of pathogenic mechanisms or disease course is yet to be determined.

In conclusion, chronic ulcerative HSV infection in HIV-infected individuals is a rare entity where diagnosis and treatment are challenging. Better understanding of the defects in HSV-specific immunity and inflammatory responses upon partial and full immune reconstitution will hopefully lead to improved management.

\section{Disclosure Statement}

The authors report no conflicts of interest. 


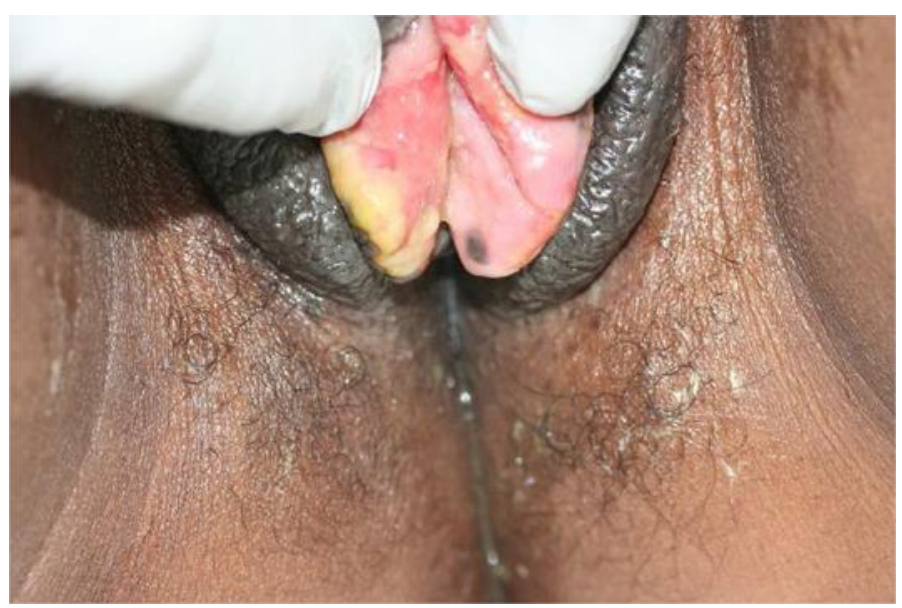

Fig. 1. Vulvar ulceration with adherent fibrinous exudates and scalloped borders.

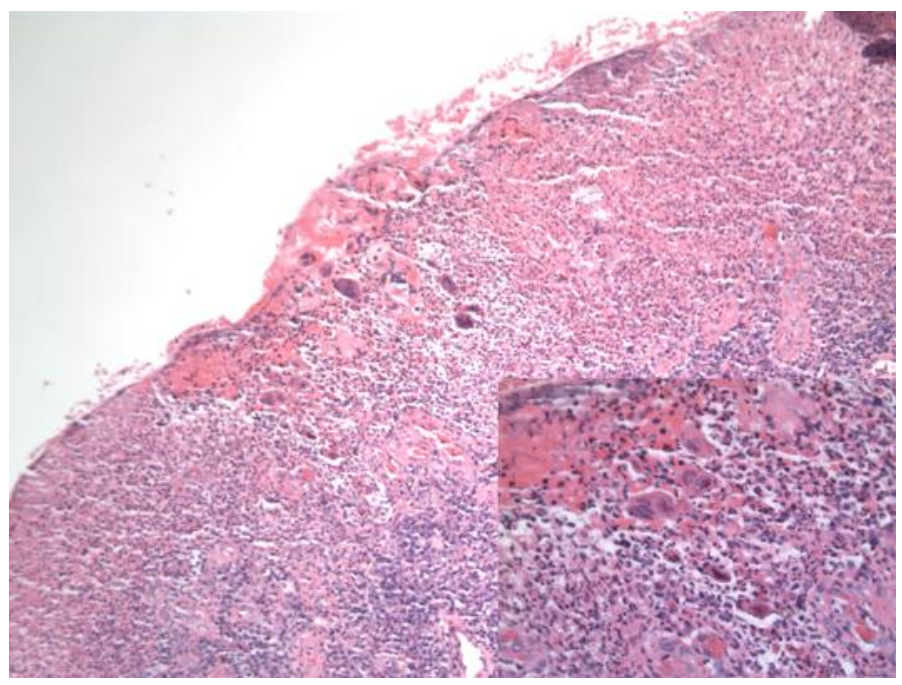

Fig. 2. Histopathology from the edge of the ulceration shows epidermal hyperplasia, mild papillary dermal edema, fibrosis in the upper lamina propria, and a diffuse, deeply extending mixed infiltrate of lymphocytes, neutrophils, and numerous eosinophils. Scattered plasma cells are also present. HE, $10 \times(40 \times$ inset $)$. 


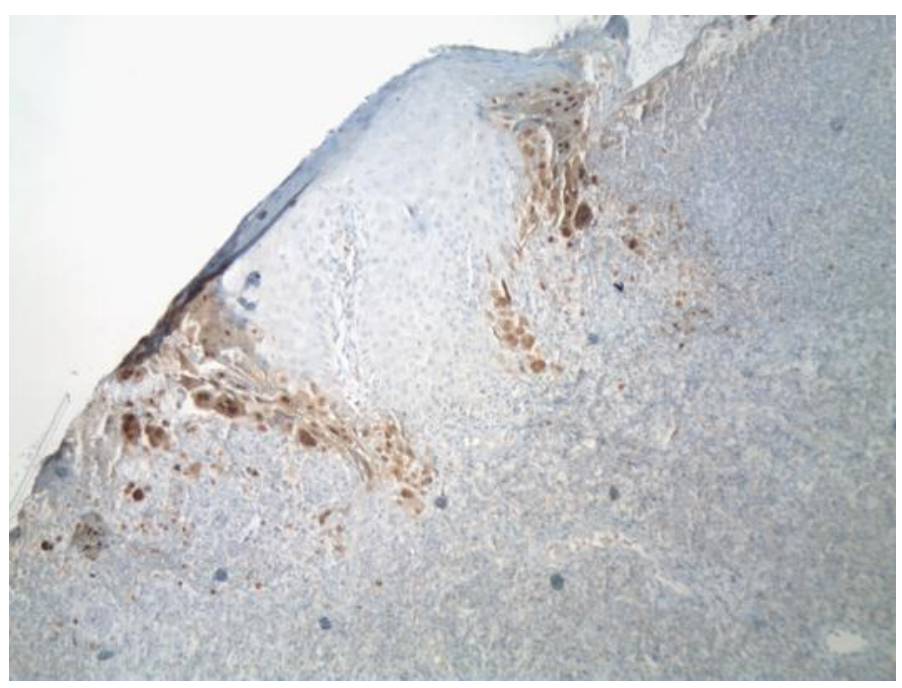

Fig. 3. Immunohistochemistry staining showing positive keratinocytes containing HSV-1/-2 antigens.

\section{References}

1 Weiss H: Epidemiology of herpes simplex virus type 2 infection in the developing world. Herpes 2004;11(suppl 1):24A-35A.

2 Strick LB, Wald A, Celum C: Management of herpes simplex virus type 2 infection in HIV type 1-infected persons. Clin Infect Dis 2006;43:347-356.

3 Couppié P, Sarazin F, Clyti E, et al: Increased incidence of genital herpes after HAART initiation: a frequent presentation of immune reconstitution inflammatory syndrome (IRIS) in HIV-infected patients. AIDS Patient Care STDs 2006;20:143-145.

4 Barde C, Piguet V, Pechère M, et al: Management of resistant mucocutaneous herpes simplex infections in AIDS patients: a clinical and virological challenge. HIV Med 2011;12:367-373.

5 Wauters O, Lebas E, Nikkels AF: Chronic mucocutaneous herpes simplex virus and varicella virus infections. J Amer Acad Dermatol 2012;66:e217-e227.

6 Centers for Disease Control: 1993 revised classification system for HIV infection and expanded surveillance case definition for AIDS among adolescents and adults. MMWR Morb Mortal Wkly Rep 1992;41:1-19.

7 Fox PA, Barton SE, Francis N, et al: Chronic erosive herpes simplex virus infection of the penis, a possible immune reconstitution disease. HIV Med 1999;1:10-18.

8 Yudin MH, Kaul R: Progressive hypertrophic genital herpes in an HIV-infected woman despite immune recovery on antiretroviral therapy. Infect Dis Obst Gyn 2008;2008:592532.

9 Lanzafame M, Mazzi R, Pace CDI, et al: Unusual, rapidly growing ulcerative genital mass due to herpes simplex virus in a human immunodeficiency virus-infected woman. Br J Dermatol 2003;149:216-217.

10 Ellis RM, Mohr MR, Oldfield III, EC, et al: Recalcitrant herpetic scrotal ulcer as a manifestation of immune reconstitution inflammatory syndrome. J Amer Acad Dermatol 2011;65:456-457.

11 Danielsen AG, Petersen CS, Iversen J: Chronic erosive herpes simplex virus infection of the penis in a human immunodeficiency virus positive man, treated with imiquimod and famciclovir. Br J Dermatol 2002;147:1034-1036.

12 Safrin S, Crumpacker C, Chatis P, et al: A controlled trial comparing foscarnet and vidaribine for aciclovir resistant mucocutaneous herpes simplex in the acquired immunodeficiency syndrome. $\mathrm{N} \mathrm{Engl} \mathrm{J} \mathrm{Med}$ 1991;325:551-555.

13 Cunningham AL, Turner RR, Miller AC, et al: Evolution of recurrent herpes simplex lesions. An immunohistochemical study. J Clin Invest 1985;75:226-233. 